\title{
ERRATUM
}

\section{Conjugation Is Not Required for Adaptive Reversion of an Episomal Frameshift Mutation in Escherichia coli}

PATRICIA L. FOSTER AND JEFFREY M. TRIMARCHI

Department of Environmental Health, Boston, University School of Public Health,

Boston University School of Medicine, Boston, Massachusetts 02118

Volume 177, no. 22, p. 6671, column 1, lines 15, 16, and 18: “ $\mu g$ " should read "mg;" Fig. 1 legend, lines 4 and 5, “ $\mu g$ " should read "mg." 